\title{
SPRAY CONE ANGLES GENERATED BY A DUAL CENTRIFUGAL INJECTOR
}

\author{
R. A. Vásquez, \\ and F. S. Costa \\ Laboratório de Combustão e Propulsão \\ Instituto Nacional de Pesquisas Espaciais \\ Rodovia Presidente Dutra, km 40 \\ CEP 12630-000, Cachoeira Paulista, SP, Brasil \\ Received: November 05, 2013 \\ Revised: December 19, 2013 \\ Accepted: January 17, 2014
}

\section{ABSTRACT}

A dual pressure swirl injector is characterized by two independent concentric chambers which can provide independent rotational levels to a single liquid or two different liquids. This paper compares theoretical, semiempirical and experimental results concerning the spray cone angles formed by injection of water, ethanol and biodiesel through a dual pressure swirl injector. Data are obtained for injection of the same liquid through the primary and secondary chambers and for injection of ethanol in the primary chamber and biodiesel through the secondary chamber of the injector. Experimental data are obtained using photographic techniques and are analyzed by an image processing software developed in Matlab language.

Keywords: dual pressure swirl injector, ethanol,biodiesel, spray cone angle.

\section{NOMENCLATURE}

A area, $\mathrm{m}^{2}$

$C_{d} \quad$ discharge coefficient

$d$ diameter, $\mathrm{m}$

K injector geometrical parameter

$l \quad$ length of the tangencial hole, $\mathrm{m}$

$\dot{m} \quad$ mass flow rate, $\mathrm{kg} / \mathrm{s}$

$n \quad$ number of holes

$P$ pressure, $\mathrm{N} / \mathrm{m}^{2}$

$r_{c v} \quad$ radius of the vortex chamber, $\mathrm{m}$

$r \quad$ radius of the tangencial hole, $\mathrm{m}$

$R \quad$ radius, $\mathrm{m}$

Re Reynolds number

$u$ axial velocity, $\mathrm{m} / \mathrm{s}$

$v$ radial velocity, $\mathrm{m} / \mathrm{s}$

$V \quad$ mean velocity, $\mathrm{m} / \mathrm{s}$

Z dimensionless parameter

\section{Greek symbols}

$\alpha \quad$ spray semi-cone angle inner chamber, ${ }^{\circ}$

$\beta \quad$ spray semi-cone angle external chamber, ${ }^{\circ}$

$\gamma \quad$ spray semi-cone angle combined, ${ }^{\circ}$

$\varepsilon \quad$ dimensionless efficiency of filling

$\lambda$ dimensionless friction coefficient

$\xi \quad$ dimensionless loss coefficient

$\psi \quad$ inclination of tangencial channels, ${ }^{\circ}$

$v \quad$ fluid kinematic viscosity, $\mathrm{m}^{2} / \mathrm{s}$

$\rho$ density, $\mathrm{kg} / \mathrm{m}^{3}$

\section{Subscripts}

$\begin{array}{ll}\text { eq } & \text { equivalent } \\ \text { inj } & \text { injector } \\ h & \text { tangential hole } \\ \text { prim } & \text { primary }\end{array}$

$\begin{array}{ll}s & \text { inner exit } \\ \text { sec } & \text { secondary } \\ o & \text { external dimension }\end{array}$

\section{INTRODUCTION}

Combustion of liquid fuels in rocket engines, gas turbines, internal combustion engines and in many industrial applications depends on the effective atomization by an injector to achieve high rates of mixing and evaporation.

A simple swirl injector consists of one or more inlets into a central vortex chamber, the inlets generally being tangential, thus providing the spin in the vortex chamber. Finally the fluid emerges from an orifice in the form of a film around the periphery of the orifice; this film then breaks into a cone of spray droplets. The spray of the liquid produced at the output of this type of injector has the approximate shape of a hollow-cone (Lefebvre, 1989).

A dual pressure swirl injector is characterized by two independent concentric vortex chambers which can provide independent rotational levels to a single liquid or two different liquids. In the case of a dual pressure swirl injector a hollow-cone is formed for each chamber and if the two cones collide there is formation of a single cone.

Generally when the cone angle is increased there is also an increase in the contact of droplets of liquid injected with ambient air, which improves the atomization process, the heat and mass transfer. However, the reduction in the cone angle improves the performance of the ignition and extends the limits of stability (Ortmann et al., 1985). The cone angle is an important external feature of a spray. Due to the interactions of the liquid fuel with air, the spray curve actually has an approximate bell shape, as shown in Fig. 1, thus presenting the difficulty of measuring the cone angle. Typically, the measurement of this 
external feature is defined as the angle $2 \alpha$ formed by two straight lines in a plane projected from the orifice discharge of injector, at a specified distance.

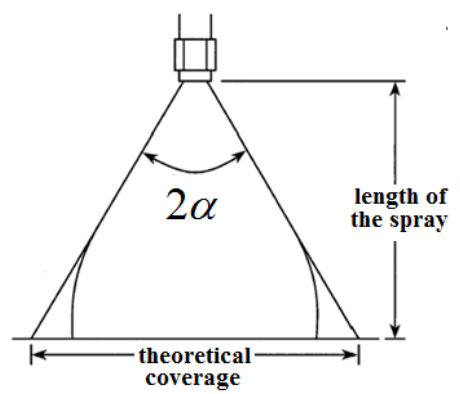

Figure 1. Definition of the cone angle.

This paper compares theoretical, semi-empirical and experimental results concerning the spray cone angles formed by injection of ethanol and soy B100 biodiesel through a dual pressure swirl injector. Data are obtained for injection of biofuels only through the primary and secondary chambers and for injection of ethanol in the primary chamber and biodiesel through the secondary chamber of the injector. Experimental data are obtained using photographic techniques and are analyzed by a graphical user interface (GUI) written in Matlab language.

\section{THEORETICAL AND SEMI-EMPIRICAL MODELS}

There are several theoretical and semi-empirical models to determine the behavior of the cone angle formed by swirl injectors.

\section{Theoretical Model}

The theoretical model used here to determine the cone angles is based on Abramovich approach, as described in detail by Vasquez (2011). Initially, it is specified the primary chamber geometric parameter:

$$
K_{\text {prim }}=\frac{\pi r_{s} R_{\text {prim }}}{A_{h}}
$$

where $r_{s}$ is the inner radius of the nozzle exit, $A_{h}$ is the area of tangential holes, $R_{\text {prim }}=r_{c v \text { prim }}-r_{\text {h prim }}$, $r_{c v \text { prim }}$ is the radius of the vortex chamber and $r_{h \text { prim }}$ is the radius of the tangential holes. Figure 2 shows that for a given $K_{\text {prim }}=K$ there is a filling efficiency, $\varepsilon_{\text {prim }}=\varepsilon$, for the nozzle exit of the primary chamber.

An equivalent discharge coefficient is determined using:

$$
C_{\text {deq prim }}=\frac{\varepsilon_{\text {eq prim }} \sqrt{\varepsilon_{\text {eq prim }}}}{\sqrt{2-\varepsilon_{\text {eq prim }}}}
$$

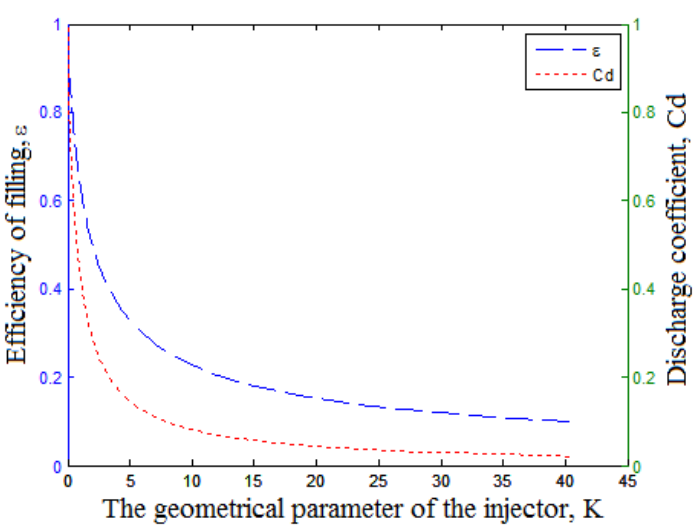

Figure 2. Behavior of geometrical parameters, the discharge coefficient and the efficiency of filling of the injector.

Then the final discharge coefficient is calculated considering the effects of geometry and friction losses:

$$
C_{d \text { final }}=\frac{C_{\text {deq prim }}}{\sqrt{1+\xi_{\text {inj }} C_{\text {deq prim }}^{2} \frac{K_{\text {prim }}^{2}}{C^{2}}}}
$$

where $C=R_{\text {prim }} / r_{s}$. The friction coefficient through the tangential channels, $\lambda=0,3164 R e^{-0.25}$, depends on the flow Reynolds number:

$$
R e=\frac{2 \dot{m}}{\pi n_{h} r_{h} \rho v}
$$

The total friction loss is computed by:

$$
\xi=\xi_{0}+\lambda \frac{l_{h}}{2 r_{h}}
$$

where the initial loss coefficient, $\xi_{0}$, is determined from Fig. 3, and the inclination of the inlet tangential channels, $\psi$, is obtained from:

$$
\psi=90^{\circ}-\tan ^{-1} \frac{r_{c v}}{l_{h}}
$$

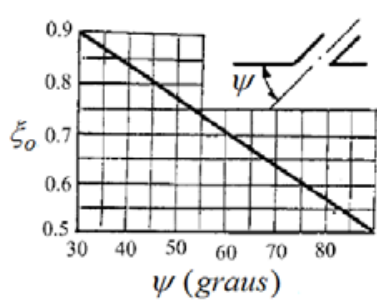

Figure 3. Initial viscous loss coefficient versus inclination of tangencial channels. 
For a given tangential inclination of the inlet channel, there is a particular $\xi_{0}$. Finally, the semicone angle of the primary chamber is given by:

$$
\sin \alpha=\frac{2 C_{d \text { final }} K_{\text {prim }} Z}{\left(1+\sqrt{1-\varepsilon_{\text {prim }}}\right) \sqrt{1-\xi_{\text {inj }} C_{d \text { final }}^{2} \frac{K_{\text {prim }}^{2}}{C^{2}}}}
$$

where $Z$ is a dimensionless parameter:

$$
Z=\frac{1}{K_{\text {prim }}} \frac{\left(1-\varepsilon_{\text {eq prim }}\right) \sqrt{2}}{\varepsilon_{\text {eq prim }} \sqrt{\varepsilon_{\text {eq prim }}}}
$$

The spray cone semi-angle in the secondary chamber is derived similarly to the primary chamber, Eq. (7), but using the following relation for the geometry of the secondary chamber:

$$
K_{\text {sec }}=\frac{\left(r_{s}^{2}-r_{\text {oprim }}^{2}\right)\left(r_{c v s e c}-r_{h s e c}\right)}{n_{h s e c} r_{h s e c}^{2}\left(r_{\text {ssec }}-r_{\text {oprim }}\right)}
$$

The value of $\varepsilon$ for the secondary chamber is obtained also using Fig. 2. In Vasquez (2011) the following relationship was obtained for the final discharge coefficient:

$$
C_{d \mathrm{sec}}=\varepsilon_{\mathrm{sec}} \sqrt{\frac{\varepsilon_{\mathrm{sec}}\left(r_{\mathrm{ssec}}^{2}-r_{\text {sprimext }}^{2}\right)}{r_{\text {ssec }}^{2}\left(2-\varepsilon_{\mathrm{sec}}\right)+r_{\text {sprimext }}^{2}\left(\varepsilon_{\mathrm{sec}}\right)}}
$$

In the case of a dual centrifugal injector, the external collision of the two spray cones formed generates another spray cone whose angle can be obtained using the momentum conservation equation. Assuming steady non viscous flow conditions, uniform pressure, uniform velocity at the exit orifices and no body forces give the equilibrium equations in the radial and axial components are:

$$
\begin{gathered}
\dot{m}_{\text {prim }} u_{\text {prim }}+\dot{m}_{\text {sec }} u_{\text {sec }}=\left(\dot{m}_{\text {prim }}+\dot{m}_{\text {sec }}\right) u_{\text {final }} \\
\dot{m}_{\text {prim }} v_{\text {prim }}+\dot{m}_{\text {sec }} v_{\text {sec }}=\left(\dot{m}_{\text {prim }}+\dot{m}_{\text {sec }}\right) v_{\text {final }}
\end{gathered}
$$

The resulting angle is defined as

$$
\gamma=\tan ^{-1}\left(\frac{\dot{m}_{\text {prim }} v_{\text {prim }}+\dot{m}_{\mathrm{sec}} v_{\mathrm{sec}}}{\dot{m}_{\text {prim }} u_{\text {prim }}+\dot{m}_{\mathrm{sec}} u_{\mathrm{sec}}}\right)
$$

and it can be written as function of cone semi-angles of inner chamber, $\alpha$, and external chamber, $\beta$ :

$$
\gamma=\tan ^{-1}\left(\frac{\dot{m}_{\text {prim }} V_{\text {prim }} \operatorname{sen} \alpha+\dot{m}_{\text {sec }} V_{\text {sec }} \operatorname{sen} \beta}{\dot{m}_{\text {prim }} V_{\text {prim }} \cos \alpha+\dot{m}_{\text {sec }} V_{\text {sec }} \cos \beta}\right)
$$

\section{Semi-empirical models}

Tanasawa and Kobayasi (Lefebvre, 1989) took into account only geometrical parameters to obtain a semi-empirical equation to calculate the spray semiangle:

$$
\alpha=90^{\circ}-\arctan \left[\frac{4}{\pi} K\left(1.37+26.9 e^{-11.1\left(\sqrt{\mathrm{A}_{\mathrm{o}}} / d_{s}\right)}\right)\right]
$$

Rizk and Lefebvre (Lefebvre, 1989) considered the effects of liquid properties, geometrical parameters, and injection pressure on the liquid film thickness, and derived the following equation for the spray angle:

$$
2 \alpha=6 K^{-0.15}\left(\frac{\Delta P d_{s}^{2} \rho}{v^{2}}\right)^{0.11}
$$

Benjamin (1998) validated an equation using a database and modified the coefficients indicated by Rizk and Lefebvre for large size injectors and obtained:

$$
2 \alpha=9.75 K^{-0.287}\left(\frac{\Delta P d_{s}^{2} \rho}{v^{2}}\right)^{0.067}
$$

\section{EXPERIMENTAL METHODOLOGY}

\section{The injector}

Figure 4 shows a scheme and Figure 5 shows a computer cut view and a photo of the dual centrifugal injector tested.

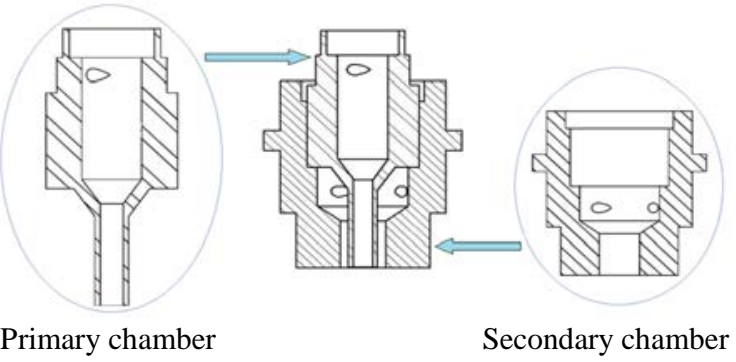

Figure 4. Scheme of the dual centrifugal injector.
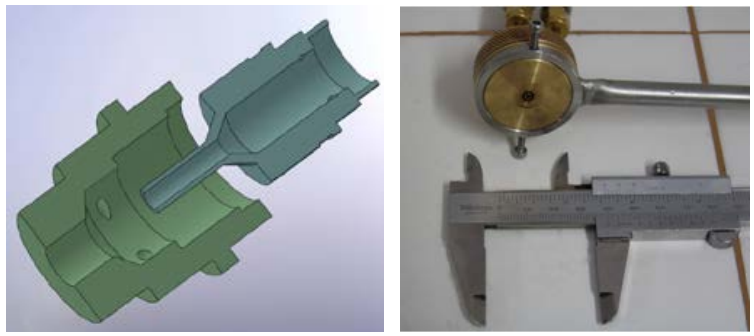

Figure 5. Cut view and photo dual of the injector. 
Table 1 shows a summary of operational and geometric parameters for design of the dual centrifugal injector, using water as the work fluid.

Table 1. Parameters of the dual centrifugal injector.

\begin{tabular}{|l|c|c|}
\hline \multicolumn{1}{|c|}{ Parameter } & $\begin{array}{c}\text { Primary } \\
\text { chamber }\end{array}$ & $\begin{array}{c}\text { Secondary } \\
\text { chamber }\end{array}$ \\
\hline Operating pressure [Pa] & $2 \times 10^{5}$ & $2 \times 10^{5}$ \\
\hline Mass flow rate [kg/s] & $10.3 \times 10^{-3}$ & $16 \times 10^{-3}$ \\
\hline Discharge coefficient & 0.1961 & 0.0922 \\
\hline $\begin{array}{l}\text { Inner diameter of the } \\
\text { outlet nozzle [m] }\end{array}$ & $1.83 \times 10^{-3}$ & $4.28 \times 10^{-3}$ \\
\hline $\begin{array}{l}\text { Number of tangential } \\
\text { channels }\end{array}$ & 2 & 4 \\
\hline $\begin{array}{l}\text { Tangencial channel } \\
\text { diameter [m] }\end{array}$ & $1 \times 10^{-3}$ & $1 \times 10^{-3}$ \\
\hline $\begin{array}{l}\text { Outer diameter of the } \\
\text { nozzle outlet [m] }\end{array}$ & $2.7 \times 10^{-3}$ & $12.5 \times 10^{-3}$ \\
\hline $\begin{array}{l}\text { Injector geometric } \\
\text { constants }\end{array}$ & 3.45 & 5 \\
\hline
\end{tabular}

\section{Physical parameters of the biofuels used}

Physical properties of ethanol and soy biodiesel B100 were measured experimentally by pycnometry to determine density, the ring method to determine surface tension and an Otswald-Cannon-Fenske viscometer to determine viscosity. Table 2 shows a summary of the physical parameters determined for the two biofuels used in this research.

Table 2. Physical-chemical properties of the biofuels.

\begin{tabular}{|c|c|c|c|}
\hline Biofuel & $\begin{array}{c}\text { Density } \\
{\left[\mathbf{k g} / \mathbf{m}^{3}\right]}\end{array}$ & $\begin{array}{c}\text { Viscosity } \\
{\left[\mathbf{N ~ s} / \mathbf{m}^{2}\right]}\end{array}$ & $\begin{array}{c}\text { Surface } \\
\text { tension } \\
{[\mathbf{N} / \mathbf{m}]}\end{array}$ \\
\hline Ethanol 96\% & 806.7 & $1.24 \times 10^{-3}$ & 0.024 \\
\hline $\begin{array}{c}\text { Soy Biodiesel } \\
\text { B100 }\end{array}$ & 875.7 & $4.88 \times 10^{-3}$ & 0.028 \\
\hline
\end{tabular}

\section{Spray angle measurement}

Figure 6 shows the experimental setup used for measuring the spray cone angle by photographic techniques. The pictures were obtained by a Sony DSC-F828 digital camera, with 8 megapixels of effective resolution, or $3264 \times 2448$ pixels. The image presented in Figure 6 shows, in the left side, the support with marks to indicate a known length to be used as a reference to relate the number of pixels and the true length of the image, allowing to determine the experimental values of the spray cone angles from the respective images.

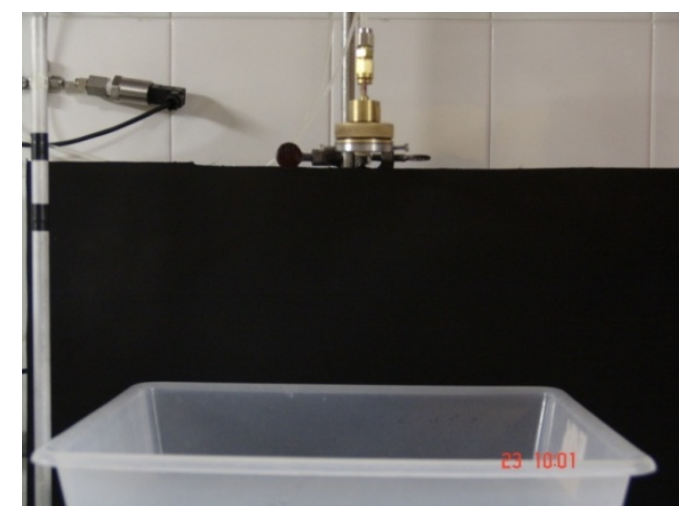

Figure 6. Experimental setup for adquisiction image.

Figure 7 shows the GUI (Graphical User Interface) developed in Matlab language, especially written for this work to process spray images. The use of this GUI is relatively simple and the images can be treated in JPEG, TIFF or BMP formats.

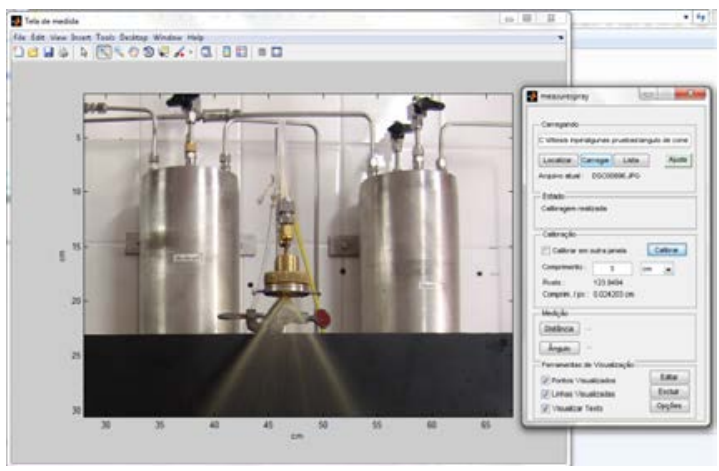

Figure 7. GUI for image processing.

After taking and selecting the appropriate images, the image processing is done with the GUI developed for this purpose, as shown in Figures 8 and 9. Finally, the experimental values of the cone angles of these images are registered. After data collection and treatment the experimental curves are obtained and compared to the theoretical data.

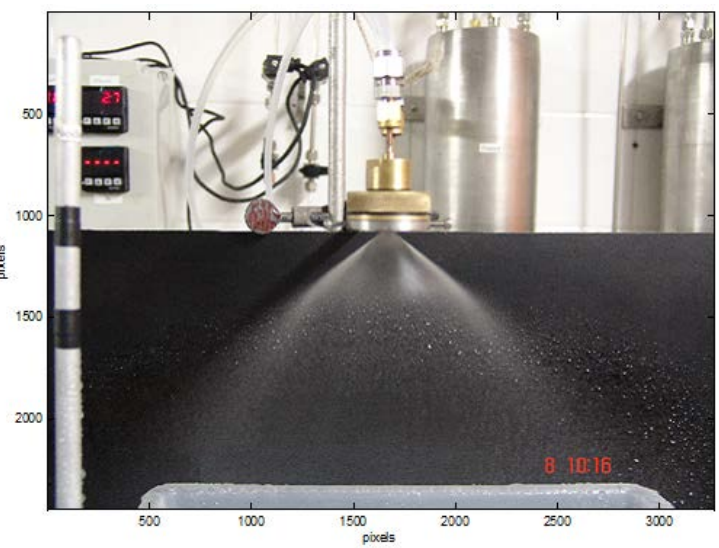

Figure 8. Not calibrated image of the cone angle measurement. 


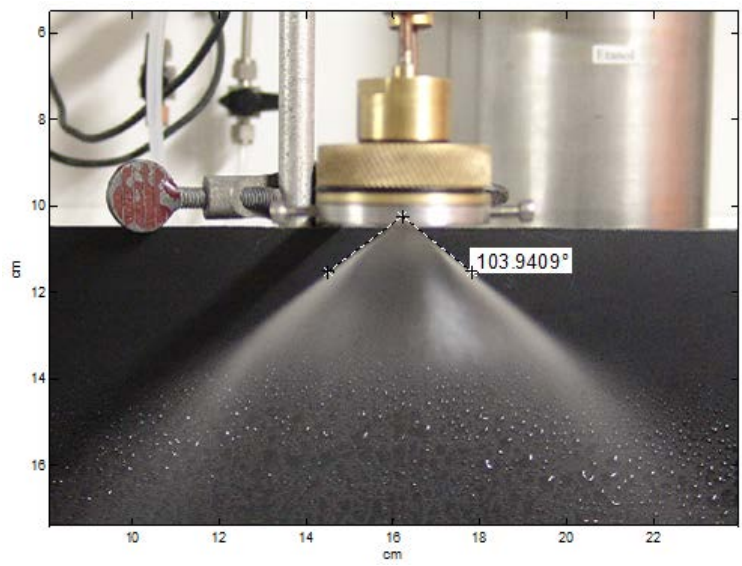

Figure 9. Calibrated image showing experimental value of the cone angle measurement.

\section{EFFECTS OF INJECTION PRESSURE ON SPRAY CONE ANGLES}

\section{Primary chamber}

Figure 10 shows theoretical, semi-empirical and experimental spray cone angles for injection of hydrated ethanol or soy B100 biodiesel in the primary chamber.
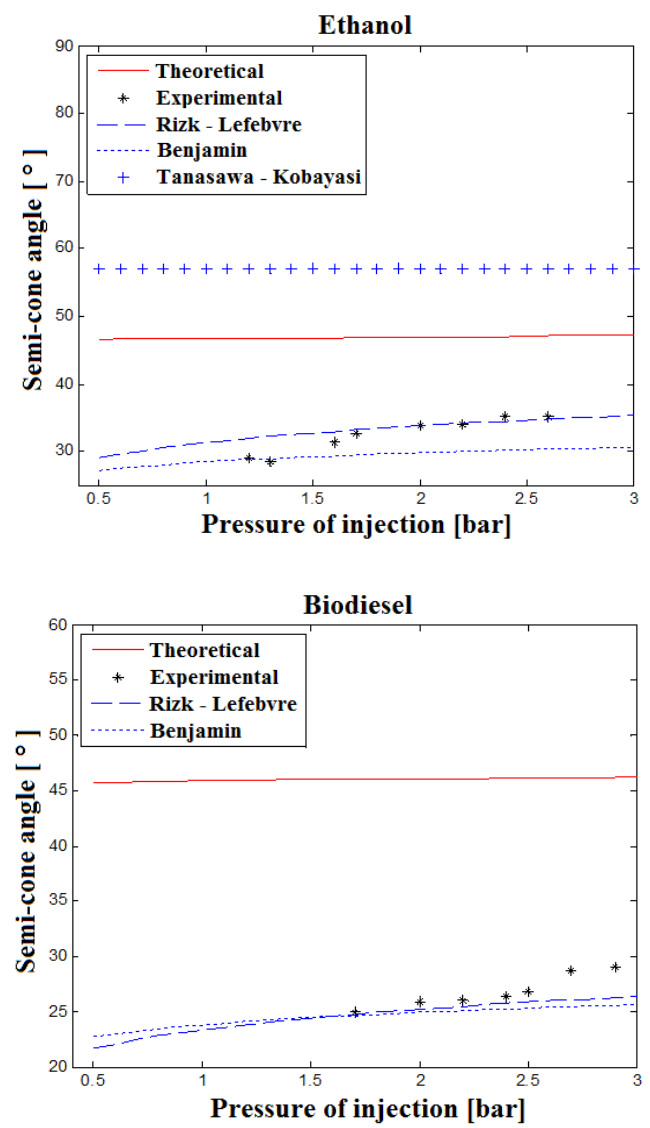

Figure 10. Cone angles generated by ethanol and soy B100 biodiesel injection in the primary chamber.

\section{Secondary chamber}

Figures 11 shows the theoretical, semi-empirical and experimental values of spray cone angles for injection of hydrated ethanol and soy B100 biodiesel in the injector secondary chamber, as a function of injection pressure.
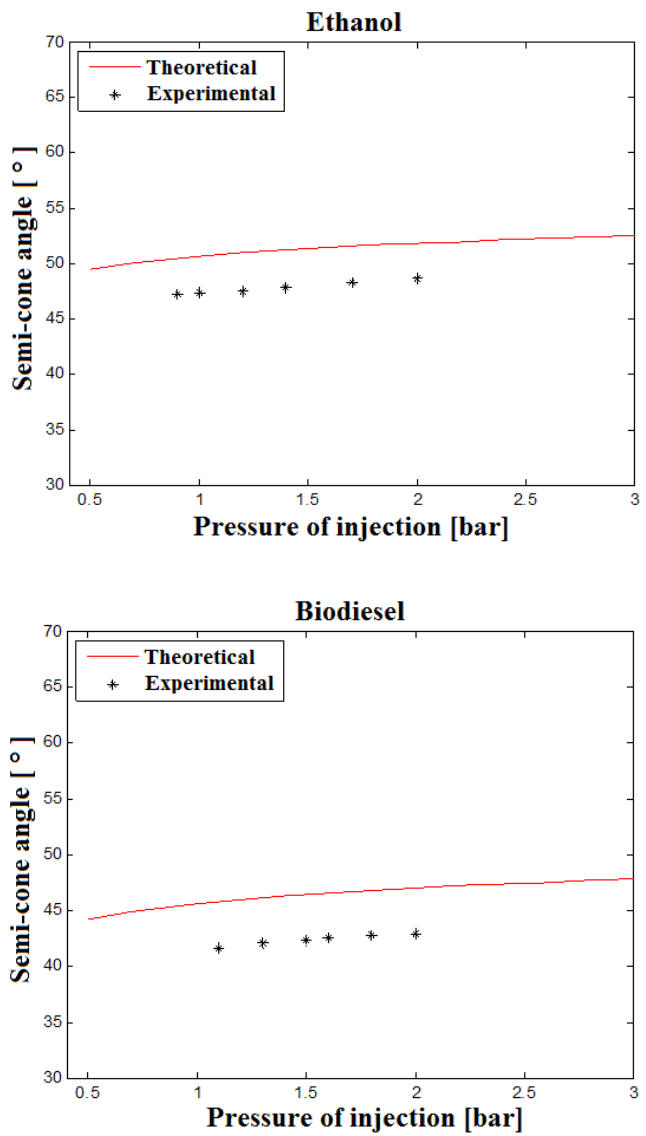

Figure 11. Cone angles generated by ethanol and soy B100 biodiesel injection in the secondary chamber.

As seen in Figs. 10 and 11, ethanol presents larger spray cone angles than soy B100 biodiesel for all pressures, in both primary and secondary chambers. There is also an increase of experimental cone angles with injection pressures (gauge pressures) for both liquids. The Risk-Lefebvre model presents the best agreement to experimental data, especially for ethanol.

\section{Mixture of ethanol and biodiesel}

Figure 12.a shows a photo of the spray formed by simultaneous injection of ethanol in the primary chamber and soy B100 biodiesel in the secondary chamber. Since the spray semi-angle for ethanol injection alone in the primary chamber varies from 27 to 35 degrees, and the spray cone angle for biodiesel injection alone in the secondary chamber varies from 42 to 43 degrees, there is no external intersection of the spray cones formed. Nevertheless, it is seen in 
Fig. 12.a that the two spray cones do interact, resulting in a combined spray cone semi-angle of about 35 degrees. This is probably caused by viscous effects and 3D fluid-dynamic effects.

Figure 12.b shows the experimental and theoretical spray cone semi-angles versus injection pressures, for simultaneous injection of ethanol in the primary chamber and soy B100 biodiesel in the secondary chamber. The theoretical values were about $30 \%$ larger than the experimental ones, probably due to neglecting viscous effects and turbulence effects.
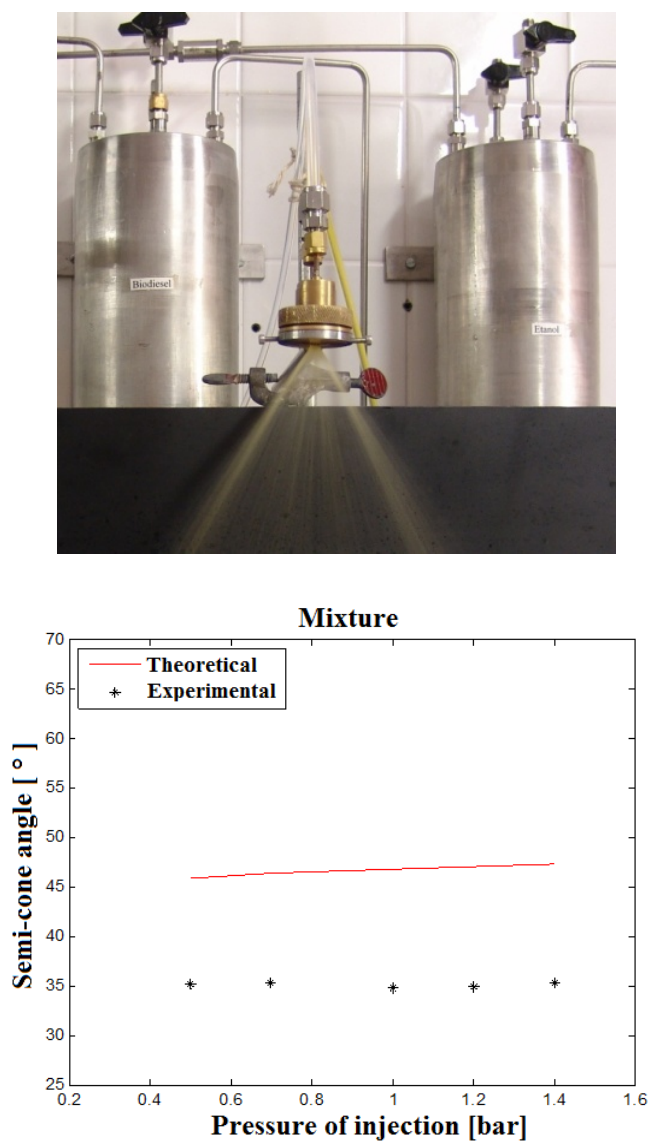

Figure 12. Experimental and theoretical spray cone angles for simultaneous injection of ethanol and biodiesel.

\section{CONCLUSIONS}

Theoretical and semi-empirical spray cone angles for injection of biofuels in a dual pressure swirl injector were calculated and compared to experimental data. A graphical user interface was developed to determine spray cone angles from spray images.

The Rizk-Lefebvre semi-empirical formulation provided the best estimates of spray cone angles for injection of ethanol or soy biodiesel B100 in the injector primary chamber, thus indicating that both liquid physical properties and injector geometry are important to determine the spray cone angles of the biofuels tested.

The interaction of spray cones was observed only for simultaneous injection of ethanol in the primary chamber and soy biodiesel B100 in the secondary chamber of the injector.

\section{ACKNOWLEDGEMENTS}

The authors acknowledge FAPESP for financial support.

\section{REFERENCES}

Ballester, J., and Dopazo, C., 1994, Discharge Coefficient and Spray Angle Measurements for Small Pressure-swirl Nozzles, Atomization and Sprays, Vol. 4, pp. 351-367.

Chen, S. K., Lefebvre, A. H., and Rollbuhler, J., 1992, Factors Influencing the Effective Spray Cone Angle of Pressure-swirl Atomizers, Journal of Engineering for Gas Turbines and Power, Vol. 114, pp. 97-103.

Ding-Yuan, L., 1987, Study and the Characteristic of the Spray Angle in Pressure Swirl Spray Atomisation, Applied Mathematics and Mechanics, Vol. 8, No. 5.

Inamura, T., Tamura, H., and Sakamoto, K., 2003, Characteristics of Liquid Film and Spray Injected from Swirl Coaxial Injector, Journal of Propulsion and Power, Vol. 19, No. 4, pp. 632-639.

Lefebvre, A. H., 1989, Atomization and Sprays, Hemisphere, New York.

Ommi, F., Nekofar, K., Kargar, A, and Movahed, E., 2009, Experimental Investigation of Characteristics of a Double-base Swirl Injector in a Liquid Rocket Propellant Engine, Leonardo Journal of Sciences, Vol. 14, pp. 92-111.

Ortmann, J., and Lefebvre, A. H., 1985, Fuel Distributions from Pressure-swirl Atomizers, Journal Propulsion and Power, Vol. 1, No. 1, pp. 11-15.

Vásquez, A. R., 2011, Development of a Dual Pressure Swirl Injector for Liquid Biofuels, Master Thesis, Instituto Nacional de Pesquisas Espaciais, Brazil. 